\title{
Efeito do laser vs LED na região do infravermelho próximo sobre a atividade muscular esquelética - estudo clínico
}

Ingrid Solange Sepúlveda Muñoz*, Leonardo Alvim Hauck, Renata Amadei Nicolau, Carlos Alberto Kelencz, Thiago dos Santos Maciel, Alderico Rodrigues de Paula Júnior

Resumo Introdução: Estudos têm demonstrado a efetividade do laser no infravermelho (IV) sobre a atividade musculoesquelética. Contudo, não foram observados estudos sobre os efeitos da radiação emitida por um LED (Light Emitting Diode) no IV sobre a atividade do músculo masseter (MM, importante músculo da mastigação), sendo este o objetivo do presente estudo. Métodos: Participaram do estudo 10 voluntários, os quais foram analisados por eletromiografia (atividade muscular, força e tempo de fadiga) e nível de lactato sanguíneo $(\mathrm{mmol} / \mathrm{L})$ após terapia placebo, laser (GaAlAs, $780 \mathrm{~nm})$ e LED (GaAlAs, $880 \mathrm{~nm})$. Os sinais obtidos previamente antes de realizadas as terapias foram tidos como controle. Os parâmetros de irradiação (laser ou LED) foram: potência de saída de $0,02 \mathrm{~W}$, densidade de energia de $4 \mathrm{~J} / \mathrm{cm}^{2}$, área do feixe de $0,2 \mathrm{~cm}^{2}$. Foram irradiados 8 pontos sobre o MM, de forma perpendicular e transcutânea, com distância de $1 \mathrm{~cm}$ entre os pontos. Resultados: A atividade muscular foi significativamente maior pós-terapia laser e LED em relação aos valores controle. Não foram encontradas diferenças significativas nos valores de força, tempos de fadiga e níveis de lactato sanguíneo pós-terapia placebo, laser ou LED, quando comparados aos valores controle. Conclusão: Os resultados obtidos no presente estudo sugerem a otimização da atividade muscular com uso de terapia laser ou LED, sem causar efeitos colaterais, como a elevação de força e aumento dos níveis de lactato.

Palavras-chave Músculo masseter, Fadiga muscular, Força muscular, Lactato, Laser, LED.

\section{Effect of laser vs LED in the near infrared region on the skeletal muscle activity - clinical study}

Abstract Introduction: Studies have demonstrated the effectiveness of the infrared laser (IR) on the skeletal muscle activity. However, it was not found studies on the effects of radiation emitted by IR LED (Light Emitting Diode) on the activity of masseter muscle (MM, important muscle of mastication), the aim of the present study. Methods: Ten volunteers were involved and their electromyography (muscle activity, strength and fatigue time) and blood lactate levels ( $\mathrm{mmol} / \mathrm{L})$ were analyzed after placebo, laser (GaAlAs, $780 \mathrm{~nm})$ and LED $(G a A l A s, 880 \mathrm{~nm})$ therapies. The signals obtained previously conducted before the therapies were taken as control. The irradiation parameters (laser or LED) were: output power of $0.02 \mathrm{~W}$, energy density of $4 \mathrm{~J} / \mathrm{cm}^{2}$ and beam area of $0.2 \mathrm{~cm}^{2}$. Eight points on the MM were irradiated in a perpendicular and transcutaneous form with a 1-cm distance between the points. Results: Muscle activity was significantly higher after laser and LED therapies when compared to control. No significant differences were found in force, fatigue time and blood lactate levels after placebo therapy, laser or LED, when compared to control. Conclusion: The results suggest the optimization of muscle activity with the use of laser or LED therapy, without causing side effects such as the increase in the force and levels of lactate.

Keywords Masseter muscle activity, Muscle fatigue, Muscle force, Lactate, Laser, LED. 


\section{Introdução}

Em se tratando de musculatura estomatognática, estudos têm abordado o emprego da terapia laser de baixa intensidade na prevenção e/ou tratamento de fadiga muscular (FM) (Califano et al., 2008, 2009; Muñoz et al., 2009; Venezian et al., 2010). A energia depositada por um laser sobre o tecido biológico pode influenciar positivamente a atividade celular (Giuliani et al., 2009; Karu et al., 2001) e o gradiente iônico transmembrana (Karu et al., 2004). A coerência (espacial) de um laser se perde nos primeiros estratos da pele (Karu, 2003), sendo que o comprimento de onda e a dose caracterizam os efeitos biológicos obtidos (Smith, 2005). Recentemente, autores observaram que a terapia LED (Light Emitting Diode) pode gerar modificação em processos metabólicos teciduais no nível do sistema musculoesquelético (Camargo et al., 2012; Paolillo et al., 2011).

Atualmente a terapia LED de baixa intensidade tem sido estudada. Segundo alguns autores seu efeito é análogo ao da terapia com laser quanto ao estímulo da microcirculação (Queiroz et al., 2008) e aumento da atividade muscular (Kelencz et al., 2010). Estudos envolvendo animais são predominantes nesta área (Camargo et al., 2012; Sussai et al., 2010), justificando estudos clínicos empregando terapia LED na região do IV. Neste sentido, o objetivo do presente estudo foi comparar o efeito da terapia laser versus terapia LED no IV sobre a atividade do músculo masseter.

\section{Métodos}

O presente estudo foi aprovado pelo Comitê de Ética do Instituto de Pesquisa \& Desenvolvimento da Universidade do Vale do Paraíba (UNIVAP), pelo número de protocolo $\mathrm{n}^{\circ}$. H01/CEP/2011. O estudo foi realizado no Centro de Laserterapia e
Fotobiologia (CEFALO) do Instituto de Pesquisa \& Desenvolvimento da UNIVAP.

\section{Casuística}

Os voluntários foram selecionados por um grupo de profissionais (co-autores do estudo) composto por dois cirurgiões dentistas, um educador físico e um fisioterapeuta, de acordo com os seguintes critérios de inclusão e exclusão:

Critérios de inclusão:

- Abertura de boca mínima de $5 \mathrm{~cm}$;

- Possuir dentição completa;

-Ausência de desordens temporomandibulares (bruxismo, luxação articular recidiva, deslocamentos, anquiloses, etc);

- Possuir boa saúde geral.

Critérios de exclusão:

- Desordens temporomandibulares;

- Processo infeccioso no sistema estomatognático;

- Limitação de abertura de boca (< que $5 \mathrm{~mm}$ );

- Dor orofacial e/ou miofascial;

- Doença neurológica;

- Deficiência auditiva;

- Uso de medicação miorrelaxante.

Os critérios de inclusão e exclusão empregados foram adotados com base em estudos prévios (Frare e Nicolau, 2008; Roldán et al., 2009; Shirani et al., 2009; Sonnesen et al., 2001). Após a seleção de voluntários a amostra foi constituída de 10 adultos do gênero masculino, caucasianos, com idade média de $28 \pm 6$ anos. Todos os voluntários foram submetidos às terapias placebo, laser e LED, respeitando-se o tempo de recuperação muscular, conforme apresentado por Celes et al. (2009), Jambassi Filho et al. (2013) e Simão et al. (2006) e seguindo o fluxograma experimental resumido na Figura 1.

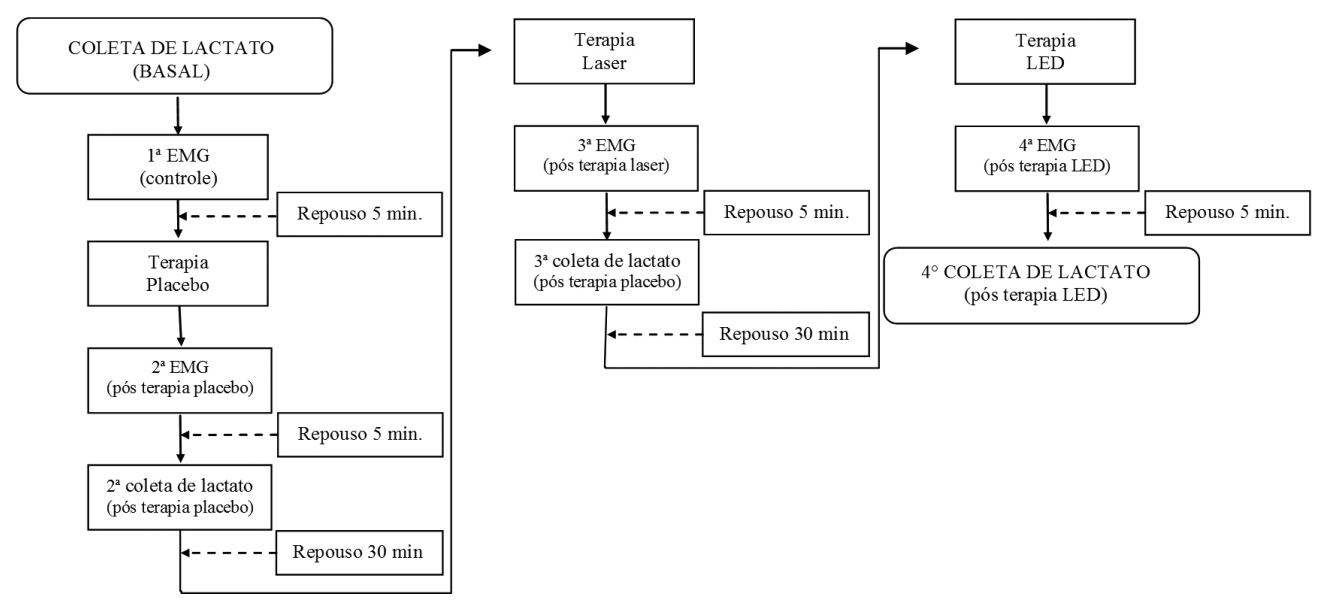

Figura 1. Fluxograma experimental.

Figure 1. Experimental flowchart. 


\section{Avaliação por lactimetria, eletromiografia e dinamometria pré e pós-terapia (placebo, laser ou LED)}

O nível de lactato sanguíneo pré-fadiga (basal) de todos os voluntários foi avaliado com o auxílio do equipamento Accusport ${ }^{\circledR}$ (Roche Diagnostics, Brasil) e tiras para análise BM-Lactate ${ }^{\circledR}$ (Roche Diagnostics, Brasil). Após a medida inicial do nível de lactato sanguíneo os voluntários receberam a fixação de eletrodos (HeartBeat, Biotronik Comercial Médica Ltda. São Paulo-SP, Brasil) para os registros eletromiográficos (Figura 2). Previamente à colocação dos eletrodos, foi realizada a tricotomia e fricção com álcool $70 \%$ sobre a pele na região do músculo masseter, com intuito de diminuir possíveis interferências na aquisição dos sinais eletromiográficos (Konrad, 2005).

Para os registros eletromiográficos foram utilizados eletrodos descartáveis de Cloreto de Prata $(\mathrm{Ag} / \mathrm{AgCl})$ revestidos por folha de papel alumínio com pré-hidrogel sintético de alta condutividade e baixa impedância (HeartBeat, Biotronik Comercial Médica Ltda. São Paulo-SP, Brasil). Para o registro eletromiográfico, utilizou-se o sistema de eletromiografia de 16 canais (EMG System do Brasil Ltda, São José dos Campos, São Paulo, Brasil), composto por eletrodos diferencias ativos (ganho de amplificação de 100 vezes), filtro analógico passa banda de 20 a $500 \mathrm{~Hz}$ e rejeição de modo comum $>120 \mathrm{~dB}$. Os sinais eletromiográficos foram amostrados com uma frequência de $1000 \mathrm{~Hz}$ (1000 amostras por segundo), digitalizados por uma placa de conversão $\mathrm{A} / \mathrm{D}$ (analógico-digital) com 16 bits de resolução, e armazenados em um computador padrão para posterior análise. Os sinais eletromiográficos são de natureza aleatória devido ao fato de que a quantidade de unidades motoras varia constantemente durante a ativação de um músculo. Para suavizar este efeito geralmente são utiizados algoritmos que calculam a média dos valores eficazes (RMS) dos sinais eletromiográficos em janelas de tempo deslizantes com duração de 20 a 500 ms. O valor eficaz é a média quadrática ou RMS (do

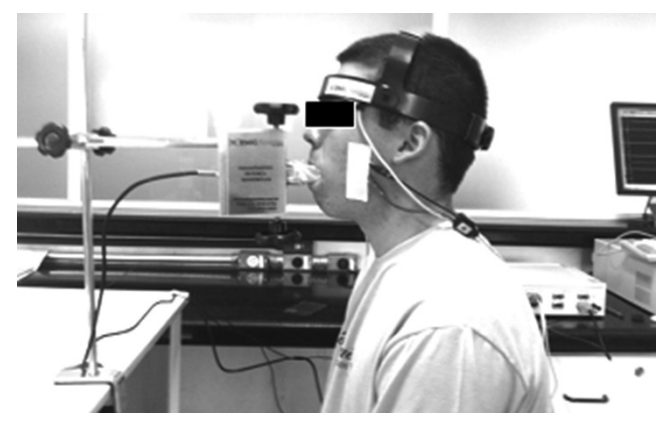

Figura 2. Voluntário realizando força máxima por 60 segundos. Figure 2. Voluntary performing maximum force for 60 seconds. inglês, Root Mean Square) que é definido como a raiz quadrada da média aritmética dos valores ao quadrado (Equação 1). Esta forma de análise não requer retificação, pois a amplitude do sinal é elevada ao quadrado (Konrad, 2005).

$R M S=\sqrt{\frac{1}{N} \sum_{i=1}^{N} x_{i}^{2}}$

O valor médio do valor RMS do sinal da atividade muscular é uma forma adequada de manipulação dos dados, pois contempla as alterações fisiológicas do sinal eletromiográfico e reflete o número médio de unidades motoras ativas em um dado intervalo de tempo (Basmajian e De Luca, 1985), permitindo uma análise do sinal. Pode-se dizer que o sinal eletromiográfico reflete diretamente o recrutamento e as características das unidades motoras detectadas de um músculo (Konrad, 2005), servindo como um indicador da atividade muscular.

Para a indução da FM foi utilizada uma plataforma oclusal (EMG System do Brasil Ltda, São José dos Campos, São Paulo, Brasil), composta por duas plataformas de aço inox, revestidas por uma borracha (atóxica) tipo silicone de $12 \mathrm{~mm}$ de largura e $4 \mathrm{~mm}$ de espessura e uma célula de carga, com capacidade de $500 \mathrm{kgf}$, para a medida da força aplicada sobre as plataformas (Figura 2). A plataforma de mordida foi inserida na cavidade bucal, entre os dentes (lado direito e esquerdo), limitando o fechamento da boca bilateralmente (dinamômetro). Sua desinfecção foi feita por lavagem, secagem, antissepsia com gaze embebida em álcool 70\% e recobrimento com filme plástico (PVC). A altura e profundidade da plataforma foram ajustadas individualmente em relação às arcadas dentárias de cada voluntário. A partir da realização de máxima intercuspidação (contração isométrica bilateral) foi possível identificar a área de maior atividade elétrica do músculo masseter para posicionar corretamente os eletrodos de registro (Perotto e Delagi, 2011). Este método preveniu o fenômeno de crosstalk, pois os músculos adjacentes ficaram afastados da área de fixação dos eletrodos (De Luca, 1997; Konrad, 2005). Um eletrodo de referência foi posicionado sobre a pele, na região da sétima vértebra cervical. Utilizando comandos verbais o voluntário era orientado a realizar a oclusão dental com força máxima, sobre a plataforma de mordida, por 60 segundos (Figura 2). Em todos os procedimentos relativos à coleta, ao registro e ao tratamento do sinal eletromiográfico, foram seguidas as recomendações da Sociedade Internacional de Eletrofisiologia e Cinesiologia (ISEK).

\section{Fototerapia}

Para os procedimentos de fototerapia foram empregados um aparelho laser GaAlAs (Twin Flex Evolution ${ }^{\circledR}$, MMOptics, São Paulo- SP, Brasil) e um aparelho de 
LED (Fisioled ${ }^{\circledR}$, MMOptics, São Paulo-SP, Brasil). A potência dos equipamentos foi medida previamente ao início dos experimentos com o auxílio de um medidor de potência (2-Watt Broadband Power/Energy Meter, 13 PEM 001/J ${ }^{\circledR}$, Melles Griot, The Netherlands).

A irradiação foi realizada em forma de contato, com o equipamento em um ângulo de $90^{\circ} \mathrm{com}$ a pele e uma leve pressão de 8 pontos sobre o músculo masseter separados a uma distância de $1 \mathrm{~cm}$ em todas as direções (Figura 3).

Cada terapia (placebo, laser ou LED) foi realizada seguindo parâmetros contidos na Tabela 1 .

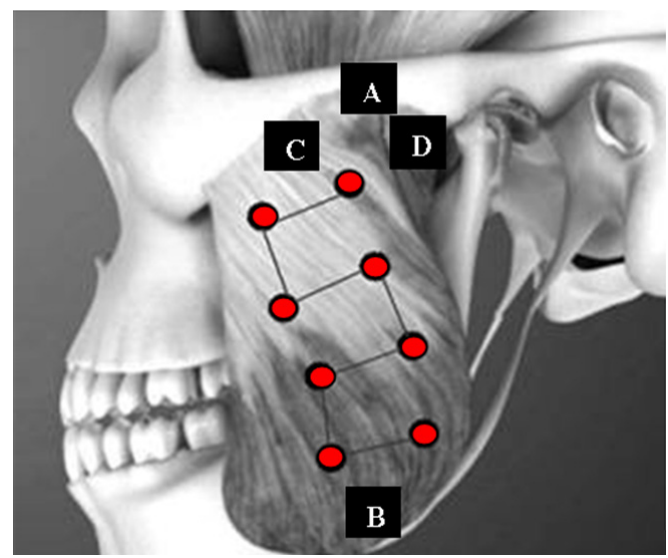

Figura 3. Pontos de terapia (placebo, laser ou LED) no músculo masseter. A - Origem (arco zigomático); B - Inserção (ângulo da mandíbula); C-Superficial: fibras que correm para baixo e ligeiramente para trás; D - Profunda: fibras que correm numa direção vertical. Adaptado de < http://www.projetohomemvirtual.com.br/downloads/ ManualHVATM.pdf $>$

Figure 3. Points of therapy (placebo, laser or LED) in the masseter muscle. A - Origin (zygomatic arch), B - Insertion (jaw angle), $C$ - Superficial: fibers running downward and slightly backward; $D$ - Deep: fibers running in a vertical direction. Adapted from http:// www.projetohomemvirtual.com.br/downloads/ManualHVATM.pdf.

Tabela 1. Parâmetros de irradiação laser e LED. A - área; DE - Densidade de energia; DP - Densidade de potência; E - Energia; $\varnothing$ - Diâmetro; P - Potência; $\mathrm{t}$ - Tempo; $\lambda$ - Comprimento de onda. Table 1. Parameters of laser and LED irradiation. A - area; DE - Energy density; DP-Power density; E-Energy; $Ø$ - Diameter; P-Power; $t$ - Time; $\lambda$ - Wavelength.

\begin{tabular}{ccc}
\hline Parâmetros & Laser & LED \\
\hline $\mathrm{A}\left(\mathrm{cm}^{2}\right)$ & 0,2 & 0,2 \\
$\mathrm{DE}\left(\mathrm{J} / \mathrm{cm}^{2}\right)$ & 4,0 & 4,0 \\
Diodo & GaAlAs & GaAlAs \\
$\mathrm{DP}\left(\mathrm{W} / \mathrm{cm}^{2}\right)$ & 0,1 & 0,1 \\
$\mathrm{E}(\mathrm{J})$ & 0,8 & 0,8 \\
$\varnothing(\mathrm{cm})$ & 0,5 & 0,5 \\
$\mathrm{P}(\mathrm{W})$ & 0,020 & 0,020 \\
$\mathrm{t}(\mathrm{s})$ & 40 & 40 \\
$\lambda(\mathrm{nm})$ & $780 \pm 5$ & $880 \pm 20$ \\
\hline
\end{tabular}

A irradiação teve início no ponto de origem (processo zigomático da maxila e arco zigomático) do músculo masseter esquerdo e terminou próxima à sua inserção (ângulo da mandíbula). Durante toda a irradiação o voluntário permaneceu com a boca fechada. O tempo de terapia (placebo, laser ou LED) foi de 40 segundos por ponto no músculo masseter esquerdo. $\mathrm{O}$ intervalo entre as terapias (placebo, laser ou LED) foi de 30 minutos (Figura 1) para cada voluntário (Celes et al., 2009; Jambassi Filho et al., 2013; Simão et al., 2006).

Uma nova coleta eletromiográfica foi realizada imediatamente após as diferentes terapias (placebo, laser ou LED). Ao final de cada terapia uma nova medida do nível de lactato sanguíneo foi realizada.

\section{Análise dos sinais}

Após a coleta dos sinais eletromiográficos, os valores de RMS foram obtidos com o auxílio do programa EMGWorks Analysis ${ }^{\circledR}$ v. 4.1 (Delsys, Boston, Massachusetts, U.S.A). O tempo total da coleta dos sinais eletromiográficos foi de 60 segundos. Para o processamento dos dados foi calculado o valor RMS da atividade eletromiográfica de cada voluntário em janelas de 0,5 segundos nos diferentes momentos experimentais (controle, placebo, laser e LED, Figura 1). Em seguida foi calculado o valor RMS médio, desprezando-se os 10 segundos iniciais e os 5 segundos finais de cada momento experimental. Os valores RMS foram normalizados para cada indivíduo dividindo-se o valor do RMS médio de cada momento experimental, pelo valor do RMS médio do controle. O mesmo procedimento foi utilizado com os dados de força.

Considerou-se o tempo de FM o instante em que a força muscular atingiu $50 \%$ do pico de força máxima (kgf) durante a contração isométrica (LopesMartins et al., 2006), obtendo-se estes dados por meio do programa EMGWorks Analysis ${ }^{\circledR}$ v. 4.1 (Delsys, Boston, Massachusetts, U.S.A).

\section{Análise estatística}

Os valores médios dos valores RMS dos sinais normalizados da atividade muscular, das forças normalizadas, bem como os tempos de fadiga (segundos) e dos níveis de lactato sanguíneo $(\mathrm{mmol} / \mathrm{L})$ foram analisados com o auxílio do programa GraphPad Prism $^{\circledR}$ v. 5.0 (GraphPad Software, Inc. La Jolla, Califórnia, E.U.A). Inicialmente foi utilizado o teste de normalidade D'Agostino para os dados de RMS, força, tempo de fadiga e lactato sanguíneo e verificou-se que todos eles satisfizeram o critério de normalidade. Com base nestes resultados foi utilizado o teste ANOVA 
e pós-teste de Tukey (Ayres et al., 2007). O nível de significância adotado foi de $5 \%(\mathrm{p}<0.05)$.

\section{Resultados}

Na Tabela 2 pode-se observar a atividade elétrica do músculo masseter esquerdo por meio dos valores RMS dos sinais das atividades musculares (valores médios) normalizados, forças normalizadas, tempos de fadiga e níveis de lactato sanguíneo pré (controle) e pós-terapia (placebo, laser ou LED). Os dados estão expressos em média \pm desvio-padrão.

A análise dos dados indica que a atividade muscular foi significativamente maior pós-terapia laser $(\mathrm{p}<0,05)$ e LED $(p<0,05)$ em relação aos valores controle, conforme apontado na Tabela 2.

Não foram encontradas diferenças significativas $(p>0,05)$ nos valores de força, tempos de fadiga e níveis de lactato sanguíneo pós-terapia placebo, laser ou LED, quando comparados aos valores controle (Tabela 2).

\section{Discussão}

Diferentes estudos clínicos têm sido realizados visando observar os efeitos da terapia com laser de baixa intensidade no IV próximo, sobre a redução de dor (Hotta et al., 2010; Shirani et al., 2009; Shinozaki et al., 2010; Venezian et al., 2010), modulação da atividade muscular (Shinozaki et al., 2010), aumento no tempo de fadiga (Almeida et al., 2012; Leal Junior et al., 2009a, 2009b), redução dos níveis de lactato sanguíneo (Leal Junior et al., 2010, 2011). Atualmente, o laser no IV tem sido empregado clinicamente sobre o músculo masseter, visando modulação terapêutica da atividade muscular (Venezian et al., 2010). Segundo Camargo et al. (2012) a terapia LED $(\lambda 940 \pm 45 \mathrm{~nm})$ pode gerar modificações em processos metabólicos em músculos gastrocnêmicos de ratos, prevenindo danos musculares e inflamações locais pós-exercício. Leal Junior et al. (2011) observaram alterações significativas dos níveis de lactato sanguíneo pósterapia com LED ( $\lambda$ 660/850 nm). Contudo, não foram observados estudos clínicos empregando terapia LED de baixa intensidade no IV próximo sobre a musculatura esquelética da face. Considerando a analogia entre as terapias laser e LED de baixa intensidade, estudos adicionais com estas ferramentas terapêuticas se fazem necessários. A investigação de indivíduos saudáveis minimiza a gama de variáveis intrínsecas que acometem o sistema estomatognático na presença de um quadro de parafuncionalidade. Assim, a amostragem investigada neste estudo baseou-se em indivíduos em homeostasia sistêmica e local.

Karu et al. (2001) demonstraram que a radiação eletromagnética (região visível e IV do espectro eletromagnético) pode ser absorvida pelo citocromo c oxidase, um fotorreceptor presente em mitocôndrias celulares. Esta absorção resulta em aumento dos níveis de adenosina trifosfato e consequentemente aumento do metabolismo. Os resultados obtidos no presente estudo demonstram um aumento da atividade muscular pós-terapia laser e LED, com a média normalizada de 1,49 e 1,56; respectivamente, em relação aos valores controle $(1,00)$. Os resultados da análise eletromiográfica apontam para o aumento da atividade elétrica e do recrutamento muscular pósfotoestimulação. Um número maior de voluntários poderia reduzir o coeficiente de variação do RMS $(\sim 39 \%)$ e lactato sanguíneo $(\sim 26 \%)$ e permitir a observação de diferenças significativas entre os grupos. Estes dados corroboram o estudo de Kelencz et al. (2010), no qual empregando radiação eletromagnética não coerente (LED $\lambda 640 \pm 40 \mathrm{~nm}$ ), verificaram aumento da atividade muscular sem alteração dos valores de força pós-terapia. De acordo com Leal Junior et al. (2010) o aumento da atividade pode culminar em aumento na resistência muscular. Segundo os autores o emprego de doses da ordem de 1 a $3 \mathrm{~J}$ pode promover a redução dos níveis de lactato sanguíneo pós-exercício intenso associado à fototerapia com laser no IV. A fadiga muscular pode ser definida como uma manifestação produzida por exercício prolongado (Dimitrova e Dimitrov, 2003), alterando a atividade muscular devido ao esgotamento de mediadores químicos (Abou-Hala et al., 2007). Os resultados não significativos do tempo de fadiga, observados no presente estudo, contrariam os dados apresentados por Almeida et al. (2012), De Marchi et al. (2012) e Leal Junior et al. (2009a, 2009b) e, os quais

Tabela 2. Valores de RMS, força, tempo de fadiga e níveis de lactato sanguíneo pré (controle) e pós-terapia (placebo, laser ou LED). Os valores de RMS e força foram normalizados em relação aos valores controle. Dados expressos em média e desvio-padrão. *p $<0,05$ vs controle. Table 2. Values of the RMS, force, fatigue time and blood lactate level before (control) and after therapy (placebo, laser or LED). The values of the RMS and force were normalized relative to control values. Data expressed as mean and standard deviation. ${ }^{*} p<0.05$ vs. control.

\begin{tabular}{lcccc}
\hline \multicolumn{1}{c}{ Parâmetros } & Controle & Placebo & Laser & LED \\
\hline RMS & $1,00 \pm 0,00$ & $1,23 \pm 0,39$ & $* 1,49 \pm 0,57$ & $1,04 \pm 0,26$ \\
Força & $1,00 \pm 0,00$ & $0,96 \pm 0,25$ & $27,56 \pm 0,72$ & $1,07 \pm 0,20$ \\
Tempo de fadiga (s) & $31,19 \pm 19,05$ & $23,09 \pm 10,30$ & 18,06 & $30,76 \pm 18,87$ \\
Lactato (mmol/L) & $2,17 \pm 0,86$ & $2,19 \pm 0,47$ & $2,40 \pm 0,50$ & $2,39 \pm 0,53$ \\
\hline
\end{tabular}


utilizaram laser na região do infravermelho próximo e energia superior às empregadas em nosso estudo. Leal Junior et al. (2009b) não observaram diferenças significativas dos níveis de lactato sanguíneo após laser terapia ( $\lambda$ de $830 \mathrm{~nm}$ ), corroborando o resultado do presente estudo, que não apontou para o aumento ou redução significativa do lactato sanguíneo pós- terapia (placebo, laser ou LED). A preservação de níveis de força próximos a valores controle, assim como a manutenção do tempo de fadiga podem preservar a integridade das fibras musculares (Kumar, 2001). Os resultados obtidos no presente estudo sugerem a otimização da atividade muscular com uso de terapia laser e LED, sem causar efeitos colaterais, como a elevação de força e aumento dos níveis de lactato.

\section{Referências}

Abou-Hala AZ, Barbosa DG, Marcos RL, Pacheco-Soares C, Silva NS. Effects of the infrared lamp illumination during the process of muscle fatigue in rats. Brazilian Archives of Biology and Technology. 2007; 50(3):403-7. http://dx.doi. org/10.1590/S1516-89132007000300006

Almeida P, Lopes-Martins RAB, Marchi T, Tomazoni SS, Albertini R, Corrêa JCF, Rossi RP, Machado GP, Silva DP, Bjordal JM, Leal ECP. Red (660 nm) and infrared (830 nm) low-level laser therapy in skeletal muscle fatigue in humans: what it better? Lasers in Medical Science. 2012; 27:453-8. http://dx.doi.org/10.1007/s10103-011-0957-3

Ayres M, Ayres M Jr, Ayres DL, Santos AAS, Ayres LL. BioEstat: Aplicações estatísticas nas áreas das ciências biomédicas. São Paulo: McGraw-Hill do Brasil; 2007. versão 5:1-380.

Basmajian JV, De Luca CJ. Muscle alive: their function revealed by electromyography. 5th ed. Baltimore: Williams \& Wilkins; 1985.

Califano AR, Kelencz CA, Nicolau RA, Muñoz ISS, Zângaro RA, Lobo PDC. Luz LED $(640 \pm 20 \mathrm{~nm})$ sobre a atividade, força e tempo de fadiga do músculo masseter - estudo clínico. Terapia Manual. 2008; 6:163-5.

Califano AR, Nicolau RA, Lobo PDC, Kelencz CA, Muñoz ISS. Electromyographic analysis of the masseter muscle and anterior bundle of the temporal muscle after muscular fatigue induction and LED irradiation $(640 \mathrm{~nm})$. Jornal Brasileiro de Laser. 2009; 2:30-8.

Camargo MZ, Frederico I, Dias L. Effects of light emitting diode (LED) therapy and cold water immersion therapy on exercise-induced muscle damage in rats. Lasers in Medical Science. 2012; 27:1051-8. http://dx.doi.org/10.1007/s10103011-1039-2

Celes R, Bottaro M, Veloso J, Ernesto C, Brown LE. Efeito do intervalo de recuperação entre séries de extensões isocinéticas de joelho em homens jovens destreinados. Revista Brasileira de Fisioterapia. 2009; 13(4):324-9.
De Luca CJ. The use of surface electromyography in biomechanics. Journal of Applied Biomechanics. 1997; 13(2):135-63.

De Marchi T, Leal EC Jr, Bortoli C, Tomazoni SS, LopesMartins RA, Salvador M. Low-level laser therapy (LLLT) in human progressive-intensity running: effects on exercise performance, skeletal muscle status, and oxidative stress. Lasers in Medical Science. 2012; 27(1):231-6. http://dx.doi. org/10.1007/s10103-011-0955-5

Dimitrova NA, Dimitrov GV. Interpretation of EMG changes with fatigue: facts, pitfalls, and fallacies. Journal of Electromyography and Kinesiology. 2003; 13:13-36. http://dx.doi.org/10.1016/S1050-6411(02)00083-4

Frare JC, Nicolau RA. Clinical analysis of the effect of laser photobiomodulation (GaAs - $904 \mathrm{~nm}$ ) on temporomandibular joint dysfunction. Revista Brasileira de Fisioterapia. 2008; 12(1):37-42.

Giuliani A, Lorenzini L, Gallamini M, Massell A, Giardino L, Calzà L. Low infra red laser light irradiation on cultured neural cells: effects on mitochondria and cell viability after oxidative stress. BMC Complementary and Alternative Medicine. 2009; 9(8):1-10. http://dx.doi.org/10.1186/14726882-9-8

Hotta PT, Hotta TH, Bataglion C, Bataglion AS, Coronatto EAS, Siéssere S, Regalo SCH. EMG analysis after laser acupuncture in patients with temporomandibular dysfunction (TMD). Implications for practice. Complementary Therapies Clinical Practice. 2010; 16:158-60. http://dx.doi.org/10.1016/j. ctcp.2010.01.002

Jambassi Filho JC, Gobbi LTB, Gurjão ALD, Gonçalves R, Prado AKG, Gobbi S. Effect of different rest intervals, between sets, on muscle performance during leg press exercise, in trained older women. Journal of Sports Science and Medicine. 2013; 12:138-43

Karu TI, Afanasyeva NI, Kolyakov SF, Pyatibrat LV, Welser L. Changes in absorbance of monolayer of living cells induced by laser irradiation at 633, 670 and $820 \mathrm{~nm}$. IEEE Journal of Quantum Electronics. 2001; 7(6):982-8. http:// dx.doi.org/10.1109/2944.983303

Karu TI. Cellular mechanism of low power laser therapy. In: Simunovic Z, editor. Lasers in Medicine and Dentistry, vol. 3. Rieka: Vitagra; 2003. p. 1-26.

Karu TI, Pyatibrat L, Afanasyeva NI. A novel mitochondrial signaling pathway activated by visibre-to-near infrared radiation. Photochemistry and Photobiology. 2004; 80:36672. http://dx.doi.org/10.1562/2004-03-25-RA-123.1

Kelencz CA, Muñoz ISS, Amorim CF, Nicolau RA. Effect of low-power gallium-aluminium-arsenium noncoherent light $(640 \mathrm{~nm})$ on muscle activity: a clinical study. Photomedicine and Laser Surgery. 2010; 28(5):647-52. http://dx.doi. org/10.1089/pho.2008.2467

Konrad P. The ABC of EMG: A practical introduction to kinesiological electromyography. version 1.0:1-60. Noraxon; Apr 2005.

Kumar, S. Theories of musculo skeletal injury causation. Ergonomics. 2001; 44:17-47. http://dx.doi. org/10.1080/00140130120716 
Leal Junior EC, Lopes-Martins RA, De Almeida P, Ramos L, Iversen VV, Bjordal JM. Effect of low-level laser therapy (GaAs 904nm) in skeletal muscle fatigue and biochemical markers of muscle damage in rats. European Journal of Applied Physiology. 2010;108(6):1083-8. http://dx.doi. org/10.2519/jospt.2010.3294

Leal Junior EC, Lopes-Martins RA, Rossi RP, De Marchi T, Baroni BM, de Godoi V, Marcos RL, Ramos L, Bjordal JM. Effect of cluster multi-diode Light Emitting Diode Therapy (LEDT) on exercise-induced skeletal muscle fatigue and skeletal muscle recovery in humans. Lasers in Surgery and Medicine. 2009a; 41(8):572-7. http://dx.doi. org/10.1002/1sm.20810

Leal Junior EC, Lopes-Martins RA, Vanin AA, Baroni BM, Grosselli D, De Marchi T, Iversen VV, Bjordal JM. Effect of $830 \mathrm{~nm}$ low-level laser therapy in exercise-induced skeletal muscle fatigue in humans. Lasers in Medical Science. 2009b; 24(3):425-31. http://dx.doi.org/10.1007/ s10103-008-0592-9

Leal Junior EC, De Godoi V, Mancalossi JL, Rossi RP, De Marchi T, Parente M, Grosselli D, Generosi RA, Basso M, Frigo L, Tomazoni SS, Bjordal JM, LopesMartins RA. Comparison between cold water immersion therapy (CWIT) and light emitting diode therapy (LEDT) in short-term skeletal muscle recovery after high-intensity exercise in athletes--preliminary results. Lasers in Medical Science. 2011; 26(4):493-501. http://dx.doi.org/10.1007/ s10103-010-0866-x

Lopes-Martins RAB, Marcos RL, Leonardo OS, Prianti AC Jr, Muscará MN, Aimbire F, Frigo L, Iversen VV, Bjordal JM. Effect of low-level laser (Ga-Al-As $655 \mathrm{~nm}$ ) on skeletal muscle fatigue induced by electrical stimulation in rats. Journal of Applied Physiology. 2006; 101:283-8. http:// dx.doi.org/10.1152/japplphysiol.01318.2005

Muñoz ISS, Kelencz CA, Nicolau RA, Sebbe TF, Pereira WG, Netto Godofredo CG. Análise eletromiográfica do músculo masseter, após indução de fadiga com aplicação de laser de baixa potência (685 e 830nm) (Resultados Preliminares). Terapia Manual. 2009; 7(31):197-201.

Paolillo FR, Milan JC, Aniceto IV, Barreto SG, Rebelatto JR, Borghi-Silva A, Parizotto NA, Kurachi C, Bagnato VS. Effects of infrared-led illumination applied during high-intensity treadmill training in postmenopausal women. Photomedicine and Laser Surgery. 2011; 29(9):639-45. http://dx.doi.org/10.1089/pho.2010.2961
Perotto A, Delagi EF. Anatomical guide for the electromyographer: the limbs and trunk. 5th ed. Springfield: Charles C. Thomas; 2011.

Queiroz LS, Wollmann DER, Nicolau RA, Pacheco MTT. Effect of LED irradiation on microcirculation of auricular mouse. Revista da APCD. 2008; 62(2):138-42.

Roldán S, Buschang PH, Isaza Saldarriaga JF, Throckmorton G. Reliability of maximum bite force measurements in age-varying populations. Journal of Oral Rehabilitation. 2009 36:801-7. http://dx.doi.org/10.1111/ j.1365-2842.2009.01997.x

Shinozaki EB, Dos Santos MBF, Okazaki LK, Marchini L, Brugnera AB Jr. Clinical assessment of the efficacy of low-level laser therapy on muscle pain in women with temporomandibular dysfunction, by surface electromyography. Brazilian Journal of Oral Sciences. 2010; 9(4):434-8.

Shirani AM, Gutknecht N, Taghizadeh M, Mir M. Low-level laser therapy and myofacial pain dysfunction syndrome: a randomized controlled clinical trial. Lasers in Medical Science. 2009; 24(5):715-20. http://dx.doi.org/10.1007/ s10103-008-0624-5

Simão R, Monteiro W, Jacometo A, Tesseroli C, Teixeira, G. A influência de três diferentes intervalos de recuperação entre séries com cargas para 10 repetições máximas. Revista Brasileira de Ciência e Movimento. 2006; 14(3):37-44.

Smith KC. Laser (and LED) therapy is phototherapy. Letter to the editor. Photomedicine and Laser Surgery. 2005; 23(1):7880. http://dx.doi.org/10.1089/pho.2005.23.78

Sonnesen L, Bakke M, Solow B. Temporomandibular disorders in relation to craniofacial dimensions, head posture and bite force in children selected for orthodontic treatment. European Journal of Orthodontics. 2001; 23(2):179-92. http://dx.doi.org/10.1093/ejo/23.2.179

Sussai DA. Low-level laser therapy attenuates creatine kinase levels and apoptosis during forced swimming in rats. Lasers in Medical Science. 2010; 25(1):115-20. http:// dx.doi.org/10.1007/s10103-009-0697-9

Venezian GC, Da Silva MAMR, Mazzetto RG, Mazzetto MO. Low level laser effects on pain to palpation and electromyographic activity in tmd patients: a double-blind, randomized, placebo-controlled study. Cranio. 2010; 28(2):84-91.

\footnotetext{
Autores

Ingrid Solange Sepúlveda Muñoz*, Leonardo Alvim Hauck, Renata Amadei Nicolau, Thiago dos Santos Maciel, Alderico Rodrigues de Paula Júnior

Centro de Laserterapia e Fotobiologia, Instituto de Pesquisa e Desenvolvimento, Universidade do Vale

do Paraíba - UNIVAP, Av. Shishima Hifumi, 2911, Urbanova, CEP 12244-000, São José dos Campos, SP, Brasil.
}

\section{Carlos Alberto Kelencz}

Laboratório de Biodinâmica, Centro Universitário Ítalo Brasileiro - UNIÍTALO, Av. João Dias , 2.046, CEP 04724-003, São Paulo, SP, Brasil. 